\title{
A new tool for simulation of geospecific multipath and obscuration of GPS / GNSS signals with relation to realistic 3-D city models
}

\author{
Karen von Hünerbein ${ }^{1}$ and Grégory Moura ${ }^{2}$ \\ ${ }^{1}$ LANGE-ELECTRONIC GmbH \\ Rudolf-Diesel-Str. 29a \\ D - 82216 Gernlinden \\ ${ }^{2}$ Oktal-SE \\ 11, avenue du Lac \\ F-31320 Vigoulet-Auzil
}

\begin{abstract}
:
Positioning and navigation with GPS and other Global Navigation Satellite Systems (GNSS) usually work well in open sky conditions like in the air and in flat landscapes. However, reception problems have been observed in mountainous and urban areas, where high structures obscure some or many Line of Sight Signals (LOS) of satellites and where the signal interacts with the local environment and multipath (MP) such as reflections, diffractions and transmissions can reach the receiver. These echoes can lead to a lack of signals and to erroneous input to the receiver front end resulting in less accurate and imprecise position fixes and sometimes to a position blackout.

For many years, GNSS hardware simulators, have been used to emulate realistic GPS, Galileo, Beidou and GLONASS signal environments and to reproduce multiple atmospheric, environmental, vehicular, antenna and satellite error effects in a controlled and repeatable way. Obscuration and multipath have been represented by different models, usually in a mathematical, stochastic, geotypical way, representing a certain type of environment but not a specific location, city map, 3D building structure or mountain profile.

One of the main obstacles was the huge wealth of data needed to define the shapes and surfaces of the structures and the physical properties of the surfaces. Another main obstacle was the processing power needed to handle both the 3D map data, the vehicle motion and the behaviour of RF signals interacting with these surfaces. In last few years, these problems have been solved by Oktal Synthetic Environment (Oktal$\mathrm{SE}$ ), who have programmed a deterministic software simulation tool capable of handling the massive amount of mapping and of calculating signal multipath and obscuration in real-time. This tool uses a GPU-oriented raytracing algorithm to compute, in record times, the masks generated by the environment as well as the impact of the multipath on the performance of the system.

For some types of GNSS receiver testing, it is useful to be able to test multipath and obscuration for specific locations and not just in a general way. Thus a new tool has been created which combines GNSS software simulation and hardware simulation by remote control, in order to enable the simulation of more realistic, geospecific obscuration and multipaths effects of GNSS signals as seen by the antenna of a moving vehicle in real-time.
\end{abstract}

Key words: GPS simulation, Ray racing, synthetic environments, multipath signals, line of sight signals, combination of test tools, remote control

\section{Introduction}

Positioning and navigation with GPS and other Global Navigation Satellite Systems (GNSS) works with very good results in open sky conditions like receivers on aircraft in flight and in flat landscapes. 8-12 GPS satellites alone are visible anywhere on Earth at any time, when the receiver has an unobstructed view to the sky, because, the GPS constellation has been fully populated with more than enough satellites, for more than a decade.

However, reception problems have been observed in mountainous and urban areas, where high structures obscure some or many Line of Sight Signals (LOS) of satellites and where the signals interact with the local environment resulting in GNSS signal reflections, called multipath (MP). In addition diffraction and transmissions can reach the receiver.

Obscuration of some or many GNSS satellites reduce the amount of signals which the receiver can use to calculate a position and in addition deteriorates the geometric distribution, resulting in higher DOP (Dilution of Position) values and thus impairing accuracy. Some of these problems can 
be solved by using 2 or more GNSS in parallel [figure 1], as using both GPS and GLONASS for example nearly doubles the amount of visible satellites and improves satellite availability in urban canyons.

\section{Effects of multipath signals on receivers:}

The receiver acquires several signals, without knowing which ones are the right ones. If the receiver uses one or several reflections for the position calculation, it calculates a solution with incorrect pseudoranges and the position fix will be less accurate. Multipath can distort the real signals by constructive and destructive interference, so that the signal strength varies, an effect that is perceived as fading [2].

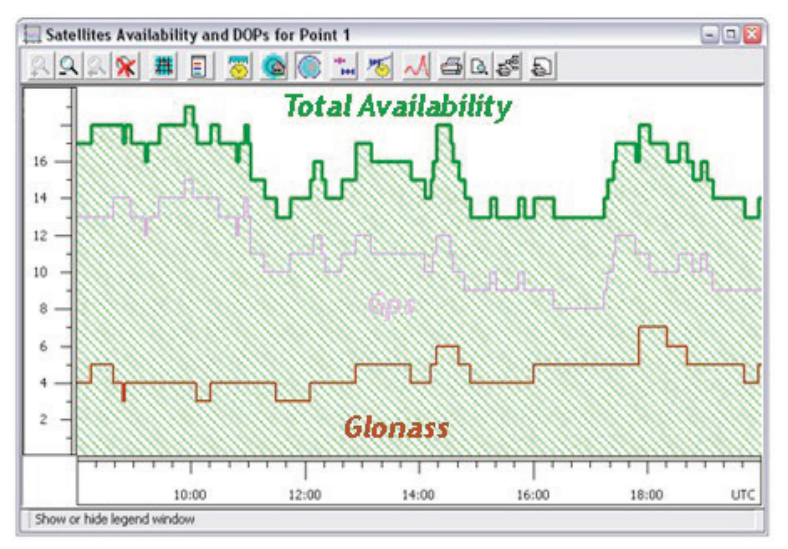

Figure 1 Satellite availability GPS and GLONASS [1]

Several multipath mitigation techniques have been described [4], among them

- large metallic ground planes

- choke ring antennas

- special receiver correlator designs

- focus on carrier phase measurements as those are less affected by multipath

A lot of work has been done to diminish the effects of multipath on the receiver by enhancing the processing algorithms. In some cases, multipath can be detected by a receiver in the presence of the Line of Sight signals, as the multipath signals take a longer route to the receiver and as they are attenuated by the reflection. In the absence of line of sight signals this comparison is not possible.

Serrano et al described a multipath mitigation technique based on a pair of antennas and mathematical models, estimating multipath with a specular reflecting raytracing algorithm [4], intended to mitigate carrier phase multipath for automated machine control. Obst et al describe an enhanced Multipath algorithm, in which a 3D environment is calculated in dynamically and then serves to detect and exclude multipath signals.

In order to test different strategies of coping with multipath and to achieve multipath resistance of receivers, RF simulators have been used for a number years generating both the GNSS line of sight signals (LOS) and multipath signals. An RF Constellation Simulator "reproduces the environment of a GNSS receiver on a dynamic platform by modelling the vehicle and satellite motion, signal characteristics, atmospheric and other effects, such that the receiver will actually navigate according to the parameters of the test scenario." [2] "By its very nature, simulation is a representation of the real world. Simulation cannot reproduce the full richness of real world conditions." [2] "... application of representative effects via simulation is proven"..." to exercise receivers and adequately identify their limitations allowing for design centring and optimisation. And, it gives complete repeatability, control and exact knowledge - down to bit level - of the signal stimulating the receiver.

This is not possible in the real world." Simulator testing is "representing the real world, rather than replicating it. Continued, successful deployment of receiver designs in many applications, prove that the simulators being used for their development and verification are accurate in their implementation of the GNSS environment." [citations from 2]

Presently RF simulators use mostly generic models, representing landscapes and buildings in a typical way to represent obscurations. Obscuration is simulated either by elevation mask angles selectable between 1 and $90^{\circ}$, by statistical models of various landscapes - such as hills far, mountains near, canyon -- by antenna patterns, by vertical planes, or by choosing a small number of simulated satellites. Multipath (MP) models employ either fixed and variable offsets or fixed mathematical models, determined by equation parameters, e.g. for sinusoidal MP.

RF simulators usually represent one signal per channel. Some complex environments with many different smooth surfaces can produce a lot of specular reflections at the same time. This means that a lot of channels are required if the receiver is located in a complex environment with many reflections. It can be very time consuming to manually determine and program all reflected signals to be adequately represented in the simulation, even for 1 location.

One effort to cope with this situation for mobile phone testing at a fixed location, was the creation of environmental masks [4,5], by subdividing the hemisphere around the receiver into little segments and allocating one of 4 categories to each segment [Figure 2]:

1) No signals 2 ) line of sight (LOS) signals only 3 ) LOS and echoes, 4) echoes only. The control software uses this mask to automatically calculate multipath and LOS signals, starting with the lowest elevation satellite. Masks can be drawn manually in the GUI see figure 3 or selected from a set of 
predefined masks.

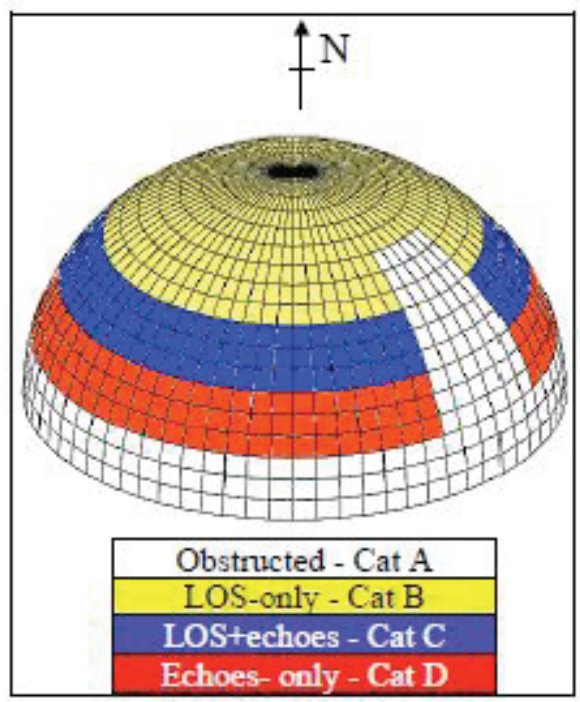

Figure 2 Concept of environmental mask from [4]

"The segments of the hemispherical mask have a 5-degree resolution. Multiple masks can be defined to represent different typical environments, such as 'Urban Canyon', 'Suburban', 'Highway', etc., and these masks can be simply selected and applied as appropriate for the particular test. The simulator calculates the arrival vector of each signal and the mask acts as a look-up-table to categorise that satellite signal for the next phase of the compromise." [citation from 4]

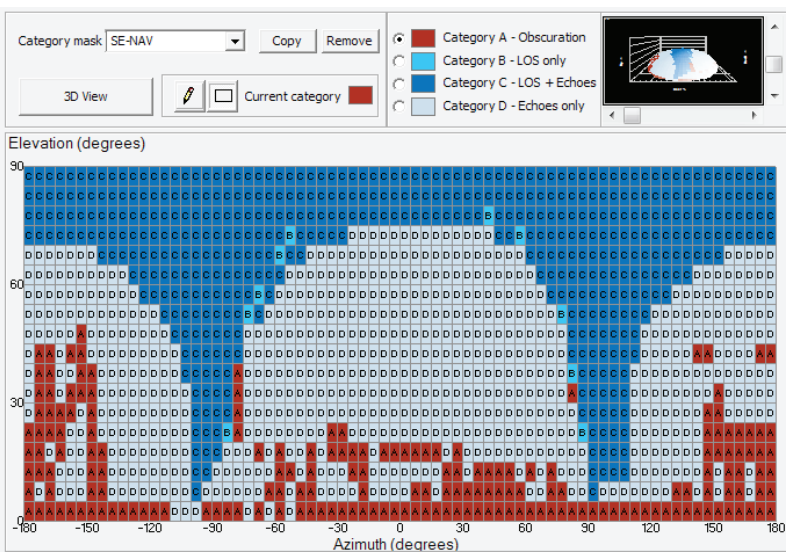

Figure 3 GUI for defining environmental mask in a signal generator control software

This approach eliminated the need to manually set every single multipath signal and switch off every obscured satellite. However, the environmental mask still limits the test to one location in a static simulation. What is needed is a dynamic test for land vehicles, with the multipath and LOS signals being calculated in real-time with relation to a 3D city / landscape map.

The aim of this work is to improve present RF simulation by incorporating obscuration and reflections specific to real world locations, such as city centers or mountain valleys, in a land vehicle or pedestrian moving along in real-time. This is achieved through a combination of a GNSS software simulator and GNSS RF hardware simulator.

\section{Overview over the solution}

- The suggested solution consists of high quality 3D maps

- $\quad$ software simulator for calculating GNSS signals

- a GNSS hardware simulator and

- a connection between software and hardware simulations either by file transfer or by remote control

\section{D environment:}

The basis for the calculation of GNSS line of sight signals and multipath signals, is a map or model of the 3 D environment (called "Synthetic Environment i.e. geometrical and physical representation of a real environment), which has to be feature rich, detailed and as close to reality as possible. Oktal-SE specializes in generation of such 3D city and terrain databases, which can be generated automatically from airborne and satellite images, from altimetry and planimetry data or from vector and raster information from other programs. Existing 3D map data available in other database formats can be imported. "Using aerial photo texture and building footprints, the mock-up significantly matches reality" [10]. The database creation tool provides editing features to modify the geometry, change attributes, like size or position, add attributes, check altimetry and more. 3 D Objects can be drawn using common software graphic tools like Trimble SketchUp ${ }^{\circledR}$ or downloaded from the internet and later inserted into the 3D environment database.

As the type of reflection strongly depends on the properties of the surface, it is necessary to distinguish between different types and textures of the surfaces, e.g. glass versus concrete, tree cover versus grass. All surfaces can be classified according to their material, their reflectivity, their temperature behaviour and absorption.

To compute and to display the GNSS LOS and multipath signals in a 3D scene, the commercially available software simulation tool SE-NAV is applied. Signals and their reflections are calculated by a deterministic GPU ray tracing algorithm, based on Geometrical Optics and Uniform Theory of Diffraction with relation to detailed 3D maps of cities and landscapes. 


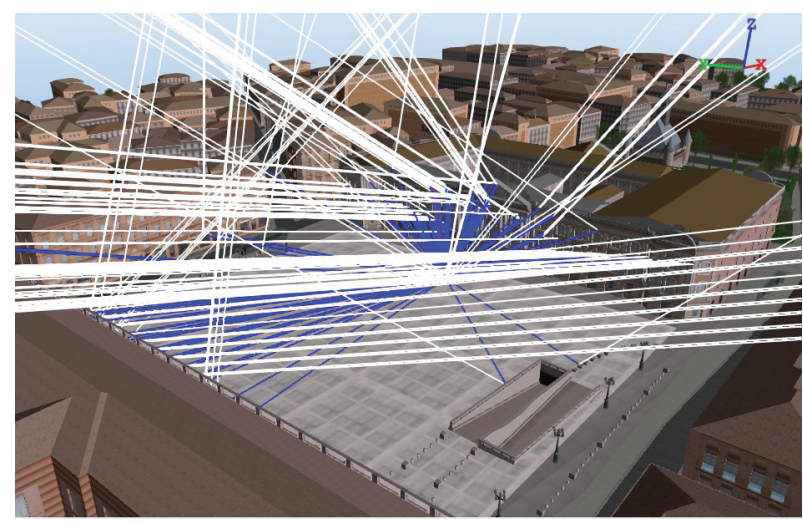

Figure $43 D$ scene modelling the centre of Toulouse with LOS (white) and multipath (blue)

In addition visibility masks, which are based on 3D masks, almanacs, antenna patterns, can be calculated, displayed and stored as . Imm files (Land Mobile Multipath Files in Spirent control software) in order to be imported in Spirent SimGEN ${ }^{\circledR}$. Visibility masks are arrays of values describing, which parts of the hemisphere around the receiver receive LOS signals, which ones are totally obscured and which parts see indirect signals like multipath or reflections [Figure 5].

The electro-magnetic parameters of the objects can be configured by the user, also the GNSS system: by import of almanacs or by building a user-defined constellation. Processing speed is high because of parallel processing in a Graphical Processing Unit (GPU). In addition, SE-NAV computes the complete link and error budgets of each transmission channel. It also calculates visibilities, DOPs and Doppler shift. Besides, two kinds of output can be generated depending on the type of simulation: output along a trajectory or coverage within an area.

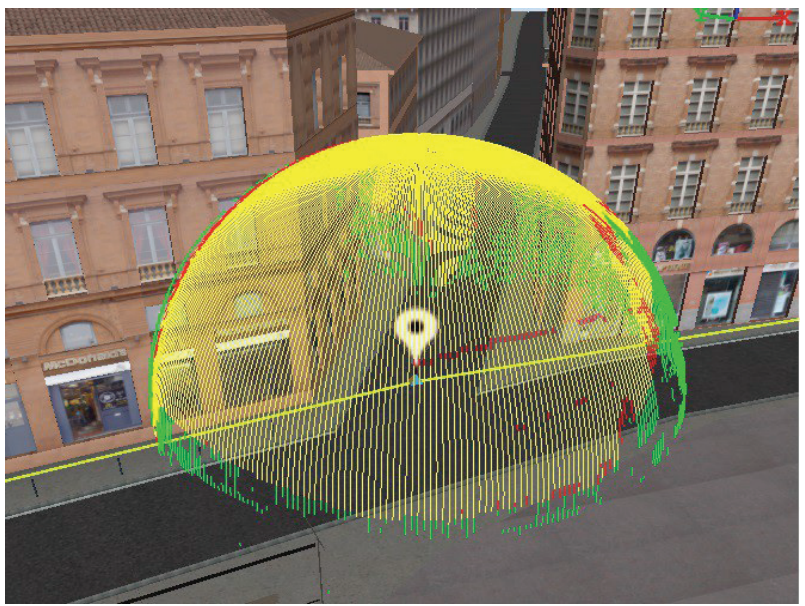

Figure 5 visibility mask of SE_NAV in Toulouse, red = LOS, yellow $=$ LOS + NLOS configuration, green $=$ NLOS = multipath, holes $=$ full obscuration

\section{Combination of the software simulator with the hardware simulator}

The software simulator provides information about obscuration and multipath to the GNSS RF simulator in one of two modes:

- offline as a set of environmental masks matching a sequence of positions along a trajectory of a land vehicle

- open loop with information containing a trajectory and a set of discrete LOS and multipath signals, encoded in remote commands and transmitted via a remote control interface. The remote control interface is available as part of the control software of the RF signal generator.

The RF signal generator generates the corresponding RF signals Line of Sight and Multipath with a rate of $1 \mathrm{~Hz}$.

\section{Status:}

A prototype is available for the offline version and the open loop version. In the future we also plan to develop a closed loop program version, with the positions of the vehicle trajectory and the satellite orbits being sent from the RF signal simulator to the software simulator and the matching signal information being sent from the SW simulator to the HW simulator.

\section{Benefits}

With this solution we have achieved a much more realistic signal environment in a GNSS RF simulation, as obscuration and multipath is now matching a 3D city map or landscape map, which is able to very accurately represent the real location. This geotypically correct signal environment is now available for real time dynamic scenarios. This improves land vehicle GNSS receiver tests in the laboratory under controlled conditions.

Calculation of the masks happens automatically, and is thus much easier and faster than manually drawing it. Thus time is saved, previously necessary for the creation of a complex scenario with multiple masks.

\section{Drawbacks}

The resulting RF simulator scenario is more realistic, but not entirely realistic. In a real environment there are moving objects, not represented by a $3 \mathrm{D}$ map. Some of the moving objects produce additional reflections, e.g. metal planes of lorries or glass of windows being opened. The quality of the simulation depends very much on the quality of the underlying $3 \mathrm{D}$ environment. The generation of a high quality, feature rich 3D scene, faithful to the geometrical and physical (i.e. EM) details, is a major task.

Each one of the environmental masks calculated along several positions of a trajectory is static, and the transitions in between positions are not represented smoothly. Vehicle motion is interpolated by the control software of the Hardware simulator, but 
the environmental masks are not interpolated in between positions.

A high number of signal channels is required for LOS signals and complex multipath signal sets in a RF hardware simulator, because each multipath signal requires a channel. Usually 12 channels are required to simulate one full GNSS constellation with all Line of Sight signals. Assuming 2 reflections per LOS signal, another 24 channels are necessary for the multipath signals alone, adding up to 36 signal channels. This number is somewhat lower, as there are fewer directly visible satellites 3D city environments.

\section{References:}

[1] http://www.gisresources.com/ Website.

[2] Spirent Communications plc, Application Note, Simulating Multipath (2011)

[3] L. Serrano, D Kim, R Langley, Multipath Minimization Method - Mitigation Through Adaptive Filtering for Machine Automation Applications, GPS World, Vol. 22, No. 7, 42-48 (2011)

[4] P. Boulton, R. Arnie, G. MacGougan, R. Kloukas, E. Cannon, G Lachapelle, Proposed Models and Methodology for Verification Testing Of A-GPS Equipped Cellular Mobile Phones in the Laboratory, ION GPS (2002)

[5] G. Lachapelle, M.E. Cannon, R. Klukas, S. Singh, R. Watson, P. Boulton, A. Read and K. Jones (2003)

Hardware Simulator Models and Methodologies for Controlled Performance Assessment of High Sensitivity AGPS Receivers. CD-ROM Proceedings of GNSS 2003, The European navigation Conference (Graz, Austria, 2225 April), Session A2 on Indoor Navigation, 21 pages.

\section{Conclusion}

We have succeeded in developing a new tool which combines GNSS software simulation and hardware simulation by remote control, in order to enable the simulation of more realistic, geospecific obscuration and multipath effects of GNSS signals as seen by the antenna of a moving vehicle in realtime. This opens up new testing possibilities for GNSS applications in traffic management and driver assistance systems.

\section{Acknowledgements:}

John Pottle Spirent Communications Plc

[6] M. Obst, S. Bauer, G. Wanielik, Urban Multipath Detection and Mitigation with dynamic $3 \mathrm{D}$ maps for Reliable Land Vehicle Localization Proceedings of the IEEE/ION PLANS., Myrtle Beach, South Carolina. (2012)

[7] D Bétaille, GNSS Accurate positioning including satellite visibility check in a multiple hypotheses 3D mapping framework, Poster, European Navigation Conference, Vienna (2013)

[8] P. Groves,L. Wang, M. Ziebart, Shadow Matching Improved GNSS Accuracy in Urban Canyons, GPS World, Feb 2012, Vol. 23, No. 2, 14-29, (2012)

[9] K. Ahmad, M. Sahmoudi, C. Macabiau, A. Bourdeau, G. Moura, Reliable GNSS Positioning in Mixed LOS/NLOS Environments Using a 3D Model, European Navigation Conference, Vienna (2013)

[10] Oktal-SE datasheet SE-AGETIM Light

[11] Oktal-SE datasheet SE-NAV 